\title{
In Addition to Insulin Resistance and Obesity, Brachial-Ankle Pulse Wave Velocity is Strongly Associated with Metabolic Syndrome in Chinese - A Population-Based Study (Taichung Community Health Study, TCHS)
}

\author{
Wen-Yuan Lin ${ }^{1,4,5}$, Ming-May Lai ${ }^{1,4}$, Chia-Ing Li ${ }^{2}$, Cheng-Chieh Lin ${ }^{1,4,6,9}$, Tsai-Chung Li ${ }^{2,7}$, \\ Ching-Chu Chen ${ }^{3,8}$, Tsann Lin ${ }^{1,4}$, and Chiu-Shong Liü ${ }^{1,4}$ \\ ${ }^{1}$ Department of Family Medicine \\ ${ }^{2}$ Medical Research \\ ${ }^{3}$ Division of Endocrinology and Metabolism, Department of Medicine, China Medical University Hospital, Taichung \\ ${ }^{4}$ Department of Family Medicine \\ ${ }^{5}$ Graduate Institute of Clinical Medicine, College of Medicine \\ ${ }^{6}$ School and Graduate Institute of Health Care Administration, College of Public Health \\ ${ }^{7}$ Graduate Institute Biostatistics \\ ${ }^{8}$ Department of Endocrinology and Metabolism, College of Chinese Medicine, China Medical University, Taichung \\ ${ }^{9}$ Institute of Health Care Administration, College of Health Science, Asia University, Taichung, Taiwan
}

\begin{abstract}
Aim: To investigate the association between arterial stiffness (present with brachial-ankle pulse wave velocity (baPWV)) and metabolic syndrome (MetS) in a population-based study of middle-aged Chinese.

Methods: MetS was defined using the AHA/NHLBI criteria. A total of 1,018 subjects aged 40 years and over were recruited in 2004. Homeostasis model assessment was applied to estimate the degree of insulin resistance (HOMA-IR). The baPWV was divided into four groups by quartiles.

Results: The prevalence of MetS and its individual components increased by the increase in baPWV quartiles. After adjusting for age, BMI, HOMA-IR, smoking, alcohol drinking, betel nut chewing, and physical activity status, multiple logistic regression revealed that baPWV groups were significantly associated with MetS. Compared with the lowest baPWV quartile, the adjusted odds ratio of having MetS in baPWV quartile II, III, IV was 2.10 (1.03-4.28), 4.48 (2.16-9.26), 10.4 (4.53-24.0) in men, and 4.20 (1.47-12.0), 14.6 (5.22-40.6), 16.3 (5.48-48.2) in women, respectively. The prevalence of MetS increased with the increase of age, HOMA-IR, and BMI groups. The optimal cut-off values of baPWV for MetS were $1,539 \mathrm{~cm} / \mathrm{sec}$ in men and $1,482 \mathrm{~cm} / \mathrm{sec}$ in women, respectively.

Conclusions: In addition to insulin resistance and obesity, baPWV was strongly related to MetS in middle-aged Taiwan Chinese. The cut-off value of baPWV for cardiovascular disease differed between genders.
\end{abstract}

J Atheroscler Thromb, 2009; 16:105-112.

Key words; Pulse wave velocity, Metabolic syndrome, Insulin resistance, Cut-off value

Address for correspondence: Chiu-Shong Liu, Department of Family Medicine, China Medical University Hospital, No 2 Yue-Der Road, Taichung, 404 Taiwan

E-mail: liucs@ms14.hinet.net

Received: July 8, 2008

Accepted for publication: October 30, 2008

\section{Introduction}

Metabolic syndrome (MetS) is recognized as a constellation of symptoms, including insulin resistance or impaired glucose metabolism, dyslipidemia, elevated blood pressure, and central obesity, which are not only associated with the subsequent development of cardiovascular diseases but also increased the risk of cardio- 
vascular disease mortality and all-cause mortality ${ }^{1-3)}$. Among these metabolic abnormalities, insulin resistance and central obesity have been recognized as the common underlying mechanism of Met ${ }^{1,4}$. Early detection and treatment of MetS had been proved to prevent further cardiovascular disease ${ }^{1)}$.

Brachial-ankle pulse wave velocity (baPWV), a non-invasive examination, reflects the stiffness of both central and peripheral muscular arteries, ${ }^{5,6}$ and serves as a simple index of the severity of arterial stiffness and atherosclerosis ${ }^{5)}$. Previous reports had proved that baPWV was associated with cardiovascular disease ${ }^{7,8)}$. Research had also found that a persistent MetS status exacerbated the severity of arterial stiffening in middle-aged Japanese men and resolution of the MetS status was associated with attenuation of the progression of vascular damage"). Yamashina et al. had proved that baPWV is a marker of atherosclerotic vascular damage and cardiovascular risk and proposed that a cut-off value of $1,400 \mathrm{~cm} / \mathrm{sec}$ serves to screen subjects, especially middle-aged subjects, of either gender ${ }^{8)}$. No appropriate cut-off value of baPWV has been proposed in Chinese. Previous studies had found that baPWV is associated with MetS in Japanese and Koreans ${ }^{10-12)}$, but not in Chinese. Furthermore, most of these studies had a small sample size and were not population-based studies, so we conducted a populationbased study in a metropolitan city in Taiwan to assess the relationship between baPWV and MetS. We then evaluate the optimal cut-off value of baPWV for diabetes, hypertension, and MetS in Taiwan Chinese.

\section{Materials and Methods}

The target population consisted of residents aged 40 and above in Taichung city, Taiwan in October, 2004. There were 363,543 residents of this area during the study, which represented about $4.09 \%$ of the national population of the same age. A two-stage sampling design was used to select residents, with the sampling rate proportional to size within each stage; 4,280 individuals were selected. During household visits, we identified 750 individuals that were ineligible and excluded them from the study sample. The reasons for exclusion included death $(n=18)$, hospitalization or imprisonment $(n=14)$, living abroad $(n=$ $39)$, moving out $(n=411)$, living in their child's house $(n=7)$, error in the sampling frame $(n=59)$, and not being at home during 3 visits made by interviewers $(n=202)$. Among 3,530 individuals selected, 2,359 agreed to participate; thus, the overall response rate was $66.8 \%{ }^{13,14)}$. Among them, 1,305 individuals who had their serum insulin level checked were selected.
Subjects with a history of cardiovascular disease or an ankle-brachial index $<0.9$ were excluded; the final population was 1,018 subjects. There was no statistical significance between insulin and non-insulin check-up groups among the body mass index (BMI), waist circumference (WC), baPWV, and the prevalence of MetS.

\section{Anthrometric Index and Laboratory Tests}

Trained staff measured height, WC, hip circumference (HipC), weight, and blood pressure as in previous reports ${ }^{13,14)}$. BMI was calculated as weight $(\mathrm{kg})$ divided by height squared $\left(\mathrm{m}^{2}\right)$, which was divided into four groups by quartiles. Blood was drawn in the morning after a 12-hour overnight fast and was sent for analysis within four hours of collection. Biochemical markers, such as total cholesterol (TCHOL), highdensity lipoprotein cholesterol (HDL-C), triglycerides, and fasting glucose level, were analyzed using a biochemical autoanalyzer (Beckman CouHer, Fullerton, CA, USA) in the Department of Clinical Laboratory (China Medical University Hospital, Taichung, Taiwan). Homeostasis model assessment was used to estimate the degree of insulin resistance [HOMA-IR = fasting insulin $\times$ fasting serum glucose $/ 22.5$, with insulin in $\mu \mathrm{U} / \mathrm{mL}$ and glucose in $\mathrm{mmol} / \mathrm{L}]$. HOMA-IR was also grouped in quartiles. Age was divided into four groups: 40-49, 50-59, 60-69, 70 years and above.

\section{Sociodemographic Factors \& Lifestyle Behaviors}

Age, gender, employment, education, diet habit, physical activity and medical history were collected by self-administered questionnaires. Smoking, alcohol drinking, and betel nut chewing history were divided into 3 classes as follows: never, former, and current. Physical activity status was divided into 2 classes: never/seldom and current.

\section{Metabolic Syndrome and Pulse Wave Velocity}

MetS was defined clinically, based on the presence of three or more of the following American Heart Association and the National Heart Lung Blood Institute (AHA/NHLBI) MetS criteria ${ }^{1)}$ : (1) central obesity (WC $\geqq 90 \mathrm{~cm}$ in men, and $\geqq 80 \mathrm{~cm}$ in women), (2) high triglycerides level $(\geqq 1.7 \mathrm{mmol} / \mathrm{L}$ or on drug treatment for elevated triglycerides), (3) low HDL-C level $(<1.03 \mathrm{mmol} / \mathrm{L}$ in men and $<1.30 \mathrm{mmol} / \mathrm{L}$ in women or on drug treatment for reduced HDL-C), (4) high blood pressure (systolic BP $\geqq 130 \mathrm{mmHg}$ or diastolic $\mathrm{BP} \geqq 85 \mathrm{mmHg}$ or under anti-hypertensive drug treatment in a patient with a history of hypertension), and (5) high fasting plasma glucose concentration $(\geqq 5.5 \mathrm{mmol} / \mathrm{L}$ or on drug treatment for ele- 
vated glucose). Diabetes was defined as fasting plasma glucose concentration $\geqq 7.0 \mathrm{mmol} / \mathrm{L}$ or on drug treatment for diabetes. Hypertension was defined as systolic BP $\geqq 140 \mathrm{mmHg}$ or diastolic $\mathrm{BP} \geqq 90 \mathrm{mmHg}$ or on drug treatment for hypertension. BaPWV was measured non-invasively with subjects in the supine position in the four limbs using a VP-1000 automated PWV/ABI analyzer (PWV/ABI; Colin Co. Ltd., Komaki, Japan $)^{5)}$. For every subject, the average of left and right baPWV was calculated for subsequent analyses. The baPWV was also divided in quartiles: $<1,330$, $1,330-1,523,1,524-1,782,>1,782 \mathrm{~cm} / \mathrm{sec}$ in men; $<1,237,1,237-1,407,1,408-1,684,>1,684 \mathrm{~cm} / \mathrm{sec}$ in women.

\section{Statistical Analysis:}

The data are presented as the means and SD unless otherwise indicated. Student's $t$-test was used to compare mean values. Log transformation was used for variables with significant deviation from normal distribution, assessed by the Kolmogorov-Smirnov test before further analyses. Analysis of variance (ANOVA) was used to compare continuous variables across baPWV quartiles. The Cochran-Armitage trend test was used to compare differences in lifestyle variables (smoking, alcohol drinking, betel nut chewing, and physical activity), the prevalence of MetS, and its individual components across baPWV quartiles. Multivariate logistic regression analysis was used to estimate the odds ratios (ORs) of MetS by age, BMI, HOMA-IR, baPWV groups, smoking, alcohol drinking, betel nut chewing, and physical activity status. One-way ANOVA was used to test the mean baPWV according to the number $(0,1,2,3,4-5)$ of components of MetS using AHA/NHLBI criteria in both genders. Receiver operating characteristic (ROC) analysis was used to compare their predictive validity, and to find their optimal cut-off values ${ }^{15,16)}$. The area under the curve (AUC) is a measure of the diagnostic power of a test. A perfect test will have an AUC of 1.0 and an $A U C=0.5$ means the test performs no better than chance. Sensitivity and specificity of the baPWV have been calculated at all possible cut-off points to find the optimal cut-off value. The optimal sensitivity and specificity were values yielding maximum sums from ROC curves. All statistical tests were 2-sided at the 0.05 significance level. ANOVA was used to evaluate the trend between baPWV and the number of MetS components. These statistical analyses were performed using the PC version of SPSS statistical software (13th version; SPSS Inc., Chicago, IL, USA).

Ethics approval for patient recruitment and data analysis was obtained from the Institutional Review
Table 1. Basic characteristic according to gender

\begin{tabular}{|c|c|c|c|}
\hline & $\begin{array}{c}\text { Men } \\
(n=486)\end{array}$ & $\begin{array}{l}\text { Women } \\
(n=532)\end{array}$ & $p$ value \\
\hline Age (years) ${ }^{\dagger}$ & $56.1 \pm 11.5$ & $53.5 \pm 9.3$ & $<0.001$ \\
\hline Height $(\mathrm{cm})^{\dagger}$ & $166.8 \pm 6.1$ & $155.5 \pm 5.4$ & $<0.001$ \\
\hline Weight $(\mathrm{kg})^{\dagger}$ & $69.2 \pm 10.4$ & $57.4 \pm 8.2$ & $<0.001$ \\
\hline BMI $\left(\mathrm{kg} / \mathrm{m}^{2}\right)^{\dagger}$ & $24.8 \pm 3.2$ & $23.8 \pm 3.2$ & $<0.001$ \\
\hline $\mathrm{WC}(\mathrm{cm})^{\dagger}$ & $86.4 \pm 8.8$ & $76.5 \pm 8.2$ & $<0.001$ \\
\hline Systolic BP $(\mathrm{mmHg})^{\dagger}$ & $134.1 \pm 18.4$ & $129.3 \pm 19.9$ & $<0.001$ \\
\hline Diastolic BP (mmHg) & $81.3 \pm 10.6$ & $74.6 \pm 11.6$ & $<0.001$ \\
\hline Heart beat $(\text { beat } / \mathrm{min})^{\dagger}$ & $67.3 \pm 9.9$ & $68.4 \pm 9.0$ & 0.029 \\
\hline Fasting glucose $(\mathrm{mmol} / \mathrm{L})^{\dagger}$ & $5.77 \pm 1.39$ & $5.56 \pm 1.40$ & 0.002 \\
\hline TCHOL (mmol/L) & $5.28 \pm 0.96$ & $5.34 \pm 0.98$ & 0.398 \\
\hline Triglycerides $(\mathrm{mmol} / \mathrm{L})^{\dagger}$ & $1.50 \pm 1.25$ & $1.11 \pm 0.72$ & $<0.001$ \\
\hline $\mathrm{HDL}-\mathrm{C}(\mathrm{mmol} / \mathrm{L})^{\dagger}$ & $1.09 \pm 0.29$ & $1.32 \pm 0.34$ & $<0.001$ \\
\hline TCHOL/HDL-C ${ }^{\dagger}$ & $5.09 \pm 1.32$ & $4.24 \pm 1.07$ & $<0.001$ \\
\hline $\operatorname{Insulin}(\mu \mathrm{U} / \mathrm{mL})^{\dagger}$ & $8.87 \pm 6.67$ & $7.76 \pm 6.60$ & 0.009 \\
\hline $\mathrm{HOMA}^{\prime} \mathrm{IR}^{\dagger}$ & $2.36 \pm 2.11$ & $2.02 \pm 2.19$ & 0.002 \\
\hline $\mathrm{baPWV}(\mathrm{cm} / \mathrm{sec})^{\dagger}$ & $1,605 \pm 388$ & $1,505 \pm 395$ & $<0.001$ \\
\hline
\end{tabular}

Abbreviations: BMI, body mass index; WC, waist circumference; HipC: hip circumference; BP, blood pressure; TCHO, total cholesterol; HDL-C, high-density-lipoprotein cholesterol; HOMA-IR: insulin resistance index by homeostasis model assessment $=$ fasting glucose $(\mathrm{mmol} / \mathrm{L}) \times$ Insulin $(\mu \mathrm{U} / \mathrm{mL}) / 22.5$; baPWV: brachial-ankle pulse wave velocity

Data are the means \pm SD. Student's $t$-test for unpaired data was used to compare mean values between genders

: Statistics were tested using log-transformed values.

Board of the China Medical University Hospital.

\section{Results}

In Table 1, we found that men had higher age, height, weight, BMI, WC, systolic BP, diastolic BP, fasting glucose, triglycerides, insulin, HOMA-IR, and baPWV than women. Table 2 shows the basic characteristics across baPWV quartiles. We found that age, BMI, WC, systolic BP, diastolic BP, fasting glucose, TCHOL, and triglycerides were statistically increased by baPWVquartiles. In Table 3, we found that the prevalence of MetS and some of its components (central obesity in women, elevated BP in both genders, elevated fasting glucose in both genders, and elevated triglycerides in women) were statistically increased by the increase of baPWV quartiles. We also found that the prevalence of MetS was statistically increased by the increase of age groups, and HOMA-IR/BMI/ baPWV quartiles (data not shown). BaPWV level was calculated according to the number of MetS components between genders. The mean baPWV level increased with an increased number of MetS compo- 
Table 2. Basic characteristic according to brachial-ankle pulse wave velocity quartile group $(n=1,018)$

\begin{tabular}{|c|c|c|c|c|c|}
\hline & $\begin{array}{c}\text { baPWV I } \\
(\mathrm{M}: 121 ; \mathrm{F}: 133)\end{array}$ & $\begin{array}{c}\text { baPWV II } \\
(\mathrm{M}: 122 ; \mathrm{F}: 133)\end{array}$ & $\begin{array}{c}\text { baPWV III } \\
\text { (M:122; F:133) }\end{array}$ & $\begin{array}{c}\text { baPWV IV } \\
\text { (M:121; F:133) }\end{array}$ & $p$ value ${ }^{*}$ \\
\hline Age (years) & $47.9 \pm 6.0$ & $51.2 \pm 8.1$ & $55.8 \pm 9.1$ & $64.1 \pm 10.5$ & $<0.001$ \\
\hline Weight (kg) & $63.4 \pm 11.5$ & $63.5 \pm 10.9$ & $63.7 \pm 11.1$ & $61.5 \pm 10.4$ & 0.073 \\
\hline BMI $\left(\mathrm{kg} / \mathrm{m}^{2}\right)$ & $23.8 \pm 3.1$ & $24.1 \pm 3.0$ & $24.6 \pm 3.4$ & $24.5 \pm 3.4$ & 0.003 \\
\hline Diastolic BP (mmHg) & $70.2 \pm 9.3$ & $75.2 \pm 9.4$ & $81.0 \pm 10.8$ & $84.8 \pm 11.3$ & $<0.001$ \\
\hline Fasting glucose $(\mathrm{mmol} / \mathrm{L})$ & $5.24 \pm 0.66$ & $5.44 \pm 1.31$ & $5.73 \pm 1.30$ & $6.22 \pm 1.88$ & $<0.001$ \\
\hline TCHOL $(\mathrm{mmol} / \mathrm{L})$ & $5.18 \pm 0.92$ & $5.32 \pm 0.98$ & $5.40 \pm 0.97$ & $5.34 \pm 1.01$ & 0.048 \\
\hline Triglycerides (mmol/L) & $1.10 \pm 0.70$ & $1.24 \pm 1.10$ & $1.43 \pm 1.12$ & $1.42 \pm 1.10$ & $<0.001$ \\
\hline $\mathrm{HDL}-\mathrm{C}(\mathrm{mmol} / \mathrm{L})$ & $1.22 \pm 0.37$ & $1.24 \pm 0.33$ & $1.19 \pm 0.33$ & $1.19 \pm 0.33$ & 0.170 \\
\hline Alcohol drinking & & & & & 0.046 \\
\hline Current & $27.2 \%$ & $26.4 \%$ & $24.7 \%$ & $19.7 \%$ & \\
\hline Former & $5.1 \%$ & $2.4 \%$ & $3.1 \%$ & $6.3 \%$ & \\
\hline Never & $67.7 \%$ & $71.3 \%$ & $72.2 \%$ & $74.0 \%$ & \\
\hline \multicolumn{6}{|l|}{ Betel nut chewing } \\
\hline Current & $3.9 \%$ & $3.9 \%$ & $2.4 \%$ & $2.8 \%$ & 0.311 \\
\hline Former & $7.1 \%$ & $6.3 \%$ & $4.3 \%$ & $6.0 \%$ & \\
\hline Never & $89.0 \%$ & $89.8 \%$ & $93.3 \%$ & $91.3 \%$ & \\
\hline \multicolumn{6}{|l|}{ Physical activity } \\
\hline Current & $61.3 \%$ & $65.1 \%$ & $66.3 \%$ & $74.0 \%$ & 0.003 \\
\hline
\end{tabular}

Abbreviations: BMI, body mass index; WC, waist circumference; BP, blood pressure; TCHO, total cholesterol; HDL-C, high-density-lipoprotein cholesterol; baPWV: brachial-ankle pulse wave velocity; M, male; F, female

*: Trend test for age, height, weight, BMI, WC, BP, fasting glucose, TCHOL, triglycerides, and HDL-C by ANOVA test. Trend test for smoking, alcohol drinking, betel net chewing, and physical activity proportion by Cochran-Armitage trend test

nents in both genders (test for trend: both $p<0.001$, Fig. 1). After adjusting for age, BMI, HOMA-IR, smoking, alcohol drinking, betel nut chewing, and physical activity status, multiple logistic regressions revealed that baPWV groups were significantly associated with MetS (Table 4). Compared with the lowest baPWV quartile, the adjusted odds ratios of having MetS for baPWV quartile II, III, IV were 2.10 (95\% confidence interval $=1.03-4.28), 4.48(2.16-9.26)$, $10.4(4.53-24.0)$ in men, and $4.20(1.47-12.0), 14.6$ (5.22-40.6), $16.3(5.48-48.2)$ in women, respectively (Table 4). The areas under the ROC curves (AUCs) of baPWV and CVD risk factors were obtained for diabetes, 0.670 in men, 0.806 in women; hypertension, 0.803 in men, 0.864 in women; MetS, 0.665 in men, 0.782 in women. AUCs were always bigger for women. The cut-off values of baPWV found to opti- mally predict diabetes, hypertension, and MetS using ROC analysis in both genders are summarized in Table 5.

\section{Discussion}

In this study, we demonstrated that the prevalence of MetS and its individual components were increased by the increase of baPWV quartiles. After adjustment for age, BMI, HOMA-IR, smoking, alcohol drinking, betel nut chewing, and physical activity status, multiple logistic regressions revealed that baPWV groups were significantly associated with MetS. The baPWV increases with an increased number of MetS components. The cut-off values of baPWV to predict diabetes, hypertension, and MetS were found to be higher than Yamashina’s suggestion. 
Table 3. The prevalence of metabolic syndrome and its individual components among brachial-ankle pulse wave velocity quartiles $(n=1,018)$

\begin{tabular}{|c|c|c|c|c|c|}
\hline & $\begin{array}{c}\text { baPWV I* } \\
\text { (M:121; F:133) }\end{array}$ & $\begin{array}{c}\text { baPWV II } \\
\text { (M:122; F:133) }\end{array}$ & $\begin{array}{c}\text { baPWV III } \\
\text { (M:122; F:133) }\end{array}$ & $\begin{array}{c}\text { baPWV IV } \\
\text { (M:121; F:133) }\end{array}$ & $p$ value $^{* *}$ \\
\hline \multicolumn{6}{|c|}{ Central obesity (\%) } \\
\hline M & 29.8 & 27.1 & 32.0 & 37.2 & 0.149 \\
\hline $\mathrm{F}$ & 17.3 & 25.6 & 39.9 & 45.1 & $<0.001$ \\
\hline \multicolumn{6}{|c|}{ Elevated BP (\%) } \\
\hline M & 24.8 & 51.6 & 76.2 & 90.9 & $<0.001$ \\
\hline $\mathrm{F}$ & 7.5 & 27.1 & 55.6 & 91.0 & $<0.001$ \\
\hline \multicolumn{6}{|c|}{ Elevated fasting glucose (\%) } \\
\hline M & 29.8 & 28.7 & 52.5 & 54.6 & $<0.001$ \\
\hline $\mathrm{F}$ & 12.0 & 24.1 & 33.1 & 47.4 & $<0.001$ \\
\hline \multicolumn{6}{|c|}{ Reduced HDL-C (\%) } \\
\hline $\mathrm{M}$ & 52.9 & 41.0 & 49.2 & 51.2 & 0.871 \\
\hline $\mathrm{F}$ & 49.6 & 48.1 & 60.2 & 56.4 & 0.095 \\
\hline \multicolumn{6}{|c|}{ Elevated triglycerides (\%) } \\
\hline M & 23.1 & 26.2 & 32.8 & 30.6 & 0.115 \\
\hline $\mathrm{F}$ & 8.3 & 10.5 & 19.5 & 17.3 & 0.008 \\
\hline \multicolumn{6}{|c|}{ Metabolic syndrome (\%) } \\
\hline M & 22.3 & 29.5 & 47.5 & 57.0 & $<0.001$ \\
\hline $\mathrm{F}$ & 4.5 & 18.0 & 43.6 & 55.6 & $<0.001$ \\
\hline
\end{tabular}

Central obesity: waist circumference $\geqq 90 \mathrm{~cm}$ in men or $\geqq 80 \mathrm{~cm}$ in women; Elevated BP (blood pressure): systolic BP $\geqq 130 \mathrm{mmHg}$ or diastolic BP $\geqq 85 \mathrm{mmHg}$ or under anti-hypertensive drug treatment in patients with a history of hypertension; Elevated fasting glucose: fasting glucose $\geqq 5.5$ $\mathrm{mmol} / \mathrm{L}$ or on drug treatment for elevated glucose; Reduced HDL-C (high-density-lipoprotein cholesterol): HDL-C $<1.03 \mathrm{mmol} / \mathrm{L}$ in men or $<1.3$ $\mathrm{mmol} / \mathrm{L}$ in women; Elevated triglycerides: triglycerides $\geqq 1.7 \mathrm{mmol} / \mathrm{L}$ or on drug treatment for elevated triglycerides; Metabolic syndrome was defined by the American Heart Association and the National Heart Lung Blood Institute criteria.

*baPWV was categorized in quartiles $(<1,330,1,330-1,523,1,524-1,782,>1,782 \mathrm{~cm} / \mathrm{sec}$ in men; $<1,237,1,237-1,407,1,408-1,684,>1,684$ $\mathrm{cm} / \mathrm{sec}$ in women)

**: using Cochran-Armitage trend test

Although the association between MetS and arterial stiffness had been proved by many studies ${ }^{11,17-20)}$, there seems to be a gender difference. For example, a previous study in Korea found that MetS is associated with baPWV in women, but not in men ${ }^{21)}$. This study also found that reduced HDL-C is not associated with PWV in both genders, similar to our study. Ferreira et al. also found that PWV increased with increasing traits of MetS in women only ${ }^{22)}$; however, Nakanishi et al. found that baPWV is strongly associated with MetS in both genders in Japanese ${ }^{23)}$. In the present study, we found that MetS is significantly associated with men as well as women. The difference between genders needs further study.

Obesity and insulin resistance are the main pathophysiologic mechanisms of Met $S^{1,4,24)}$. In this study, we found that age, BMI, and insulin resistance (present with HOMA-IR) were all significantly associated with MetS in both genders. With the increase of age group, BMI, insulin resistance, and baPWV quartiles, the prevalence of MetS also increased in our study,

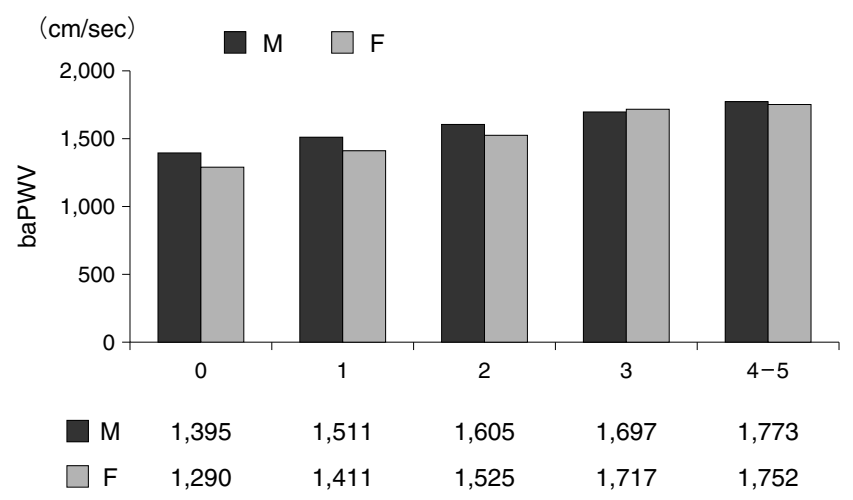

Fig. 1. Mean baPWV according to the number $(0,1,2,3,4-$ 5) of components of metabolic syndrome using AHA/ NHLBI criteria for both genders (ANOVA test, test for trend, both $p<0.001$ ).

Mean baPWV among 0, 1, 2, 3, 4-5 of the components of metabolic syndrome were $1,395 \pm 213,1,511 \pm 375,1,605 \pm 359,1,697$ $\pm 430,1,773 \pm 394 \mathrm{~cm} / \mathrm{sec}$ in men and $1,290 \pm 206,1,411 \pm 348$, $1,525 \pm 460,1,717 \pm 396,1,752 \pm 329 \mathrm{~cm} / \mathrm{sec}$ in women, respectively.

Test for trend: both $p<0.001$ 
Table 4. Adjusted odds ratios ${ }^{\#}$ (95\% confidence interval) of having metabolic syndrome using AHA/NHLI definitions derived from multiple logistic regression analysis using age, BMI, HOMA-IR, and baPWV quartiles as independent variables, adjusted for smoking, alcohol drinking, betel nut chewing, and physical activity status

\begin{tabular}{lll}
\hline & \multicolumn{1}{c}{ Men $($ MetS) } & \multicolumn{1}{c}{ Women $(\mathrm{Met} S)$} \\
\hline Age (years) & $1.00(0.97-1.02)$ & $1.04(1.01-1.07)^{*}$ \\
BMI $\left(\mathrm{kg} / \mathrm{m}^{2}\right)$ & $1.44(1.30-1.59)^{* * *}$ & $1.36(1.24-1.49)^{* * *}$ \\
HOMA-IR $^{* * *}$ & $1.41(1.21-1.63)^{* * *}$ & $1.35(1.13-1.60)^{* *}$ \\
baPWV I $^{\dagger}$ & 1.00 (Reference) $^{*}$ & $1.00($ Reference) \\
baPWV II & $2.10(1.03-4.28)^{*}$ & $4.20(1.47-12.0)^{* *}$ \\
baPWV III & $4.48(2.16-9.26)^{* * *}$ & $14.6(5.22-40.6)^{* * *}$ \\
baPWV IV & $10.4(4.53-24.0)^{* * *}$ & $16.3(5.48-48.2)^{* * *}$ \\
\hline
\end{tabular}

Abbreviations: MetS, metabolic syndrome; BMI, body mass index; AHA/NHLBI: American Heart Association/National Heart Lung Blood Institute; HOMA-IR: insulin resistance index by homeostasis model assessment $=$ fasting glucose $(\mathrm{mmol} / \mathrm{L}) *$ Insulin $(\mu \mathrm{U} / \mathrm{mL}) / 22.5$; baPWV: brachial-ankle pulse wave velocity;

${ }_{p}^{*}<0.05,{ }^{* *} p<0.01,{ }^{* * *} p<0.001$;

\#: Adjusted odds ratio: adjustment for age, BMI, HOMA-IR, smoking, alcohol drinking, betel nut chewing, and physical activity status.

${ }^{\dagger}$ baPWV was categorized in quartiles $(<1,330,1,330-1,523,1,524-$

$1,782,>1,782 \mathrm{~cm} / \mathrm{sec}$ in men; $<1,237,1,237-1,407,1,408-1,684$,

$>1,684 \mathrm{~cm} / \mathrm{sec}$ in women)

similar to other studies ${ }^{4,24,25)}$. Another study in Japan found that an increased number of MetS components was highly correlated with increased baPWV. The author concluded that the underlying mechanism was probably due to insulin resistance ${ }^{11)}$. As we know that age is one of the major determinants of baPWV, in the present study, we proved that baPWV was not only associated with MetS after adjustment for obesity, insulin resistance, age, and other lifestyle factors but was also associated with the number of MetS components. Blood pressure is another major determinant of baPWV ${ }^{20)}$, but hyperglycemia, central obesity, and dyslipidemia were the traits associated with arterial stiffness ${ }^{17)}$. In addition to high blood pressure, however, central obesity, dyslipidemia, and hyperglycemia were all significantly associated with baPWV in our study. Except for obesity and insulin resistance, the linkage between baPWV and MetS may be through the inflammation response. Research had found that the chronic inflammation response induced endothelial cell dysfunction, and progressed to atherosclerosis, and then caused cardiovascular disease ${ }^{26,27)}$. In the present study, we found that the level of high-sensitivity C-reactive protein (hs-CPR) increased with an increased number of MetS components and the baPWV quartiles (data not shown). Inflammation (assessed by hs-CPR) may be the underlying mechanism between baPWV and MetS.

An appropriate cut-off value of baPWV was proposed by Yamashina $e t$ al. ${ }^{8}$. They found that a cut-off value of $1,400 \mathrm{~cm} / \mathrm{sec}$ was enough to detect atherosclerotic vascular damage and cardiovascular risk in middle-aged Japanese. In our study, however, the optimal cut-off values of baPWV for MetS were higher than Yamashina's suggestion. The optimal cut-off value of baPWV for MetS value is around $1,540 \mathrm{~cm} / \mathrm{sec}$ in men and 1,480 cm/sec in women, higher than Yamashina's suggestion and showing a gender difference. The mean values of baPWV (adjusted for age, smoking

Table 5. Optimal cut-off values, sensitivities and specificities for baPWV predictive of CVD risk factors

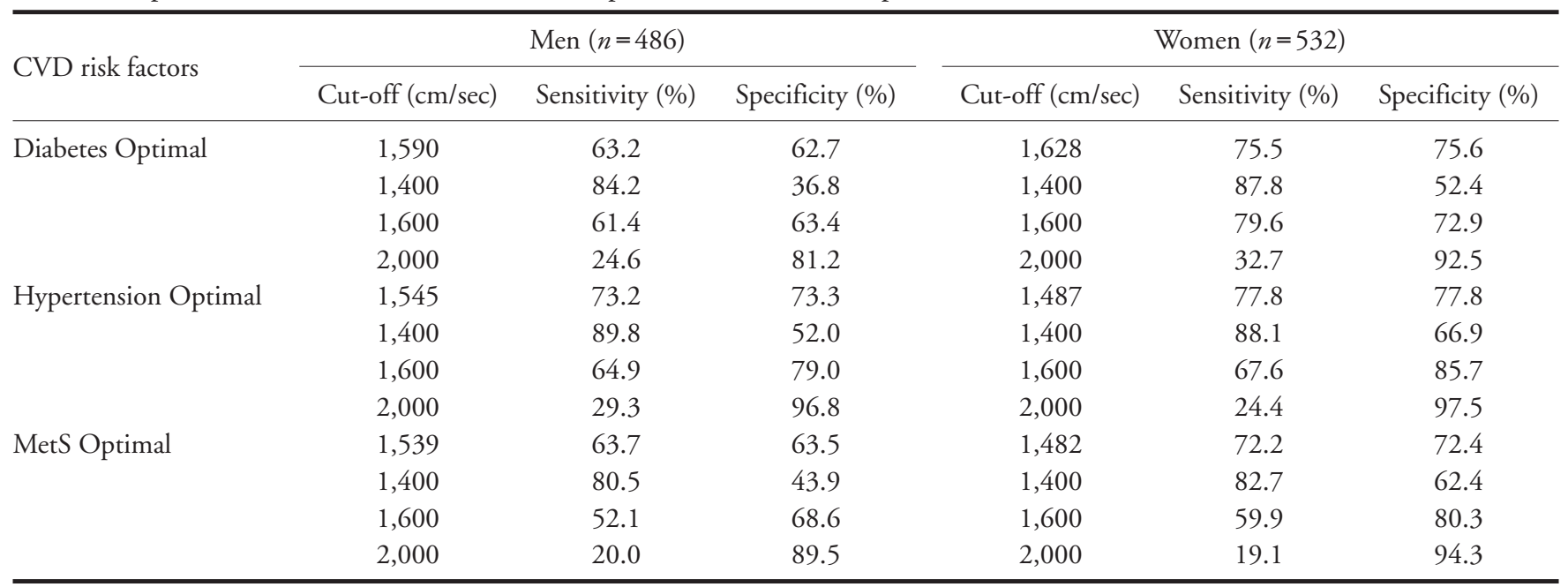

Abbreviations: CVD: cardiovascular disease; baPWV: brachial-ankle pulse wave velocity; MetS, metabolic syndrome 
and alcohol drink status) for MetS ( $\geqq 3 \mathrm{Met} S$ components) reported by Nakanishi were $1,665 \mathrm{~cm} / \mathrm{sec}$ in men and $1,661 \mathrm{~cm} / \mathrm{sec}$ in women in Japanese ${ }^{23)}$. In our study, the mean values of baPWV (adjusted for age, smoking and alcohol drink status) for MetS ( $\geqq 3$ components of MetS) were $1,728 \mathrm{~cm} / \mathrm{sec}$ in men and $1,729 \mathrm{~cm} / \mathrm{sec}$ in women, respectively (data not shown). From these data, it can be seen that the cut-off value of baPWV $(1,400 \mathrm{~cm} / \mathrm{sec})$ for cardiovascular disease is not appropriate. Further longitudinal study is necessary to clarify the appropriate cut-off value of baPWV in Asian populations.

There are some limitations of the study. First, this is a cross-section study; we do not have the exact events of cardiovascular disease, so we can not prove the causality between baPWV and MetS; however, we found a close association between baPWV and MetS as well as the MetS components, which are all risk factors of cardiovascular disease. Further prospective study is therefore necessary. Secondly, the population is from a metropolitan city in Taiwan, and may not suitable to be applied for rural areas in Taiwan as well as other countries; however, many other similar studies support our hypothesis. Differences between race and gender should be studied in the future.

In conclusion, baPWV was associated with risk factors of cardiovascular disease in the present study. The optimal cut-off values of baPWV for MetS were found to be different in the previous study, which should be taken as a screening value to detect cardiovascular disease in Chinese. BaPWV measurements can be used as a routine clinical examination in the general population to detect MetS, which is a major cardiovascular disease in Taiwan Chinese. This merits further study.

\section{Grant Support}

This study was supported by grants from the National Science Council of Taiwan (NSC93-2314B-039-025 and NSC 94-2314-B-039-024)

\section{References}

1) Grundy SM, Cleeman JI, Daniels SR, Donato KA, Eckel RH, Franklin BA, Gordon DJ, Krauss RM, Savage PJ, Smith SC Jr, Spertus JA, Costa F; American Heart Association; National Heart, Lung, and Blood Institute: Diagnosis and management of the Metabolic Syndrome: an American Heart Association/National Heart, Lung, and Blood Institute Scientific Statement. Circulation, 2005; 112:2735-2752

2) Wilson PW, D'Agostino RB, Parise H, Sullivan L, Meigs JB: Metabolic syndrome as a precursor of cardiovascular disease and type 2 diabetes mellitus. Circulation, 2005; 112:3066-3072

3) Lakka HM, Laaksonen DE, Lakka TA, Niskanen LK, Kumpusalo E, Tuomilehto J, Salonen JT: The metabolic syndrome and total and cardiovascular disease mortality in middle-aged men. JAMA, 2002; 288:2709-2716

4) Lin WY, Yang WS, Lee LT, Chen CY, Liu CS, Lin CC, Huang KC: Insulin resistance, obesity, and metabolic syndrome among non-diabetic Pre- and Post-menopausal women in North Taiwan. Int J Obes (Lond), 2006; 30:912-917

5) Yamashina A, Tomiyama $H$, Takeda $K$, Tsuda $H$, Arai $T$, Hirose K, Koji Y, Hori S, Yamamoto Y: Validity, reproducibility, and clinical significance of noninvasive brachial-ankle pulse wave velocity measurement. Hypertens Res, 2002; 25:359-364

6) Tomiyama H, Yamashina A, Arai T, Hirose K, Koji Y, Chikamori T, Hori S, Yamamoto Y, Doba N, Hinohara S: Influences of age and gender on results of noninvasive brachial-ankle pulse wave velocity measurement--a survey of 12517 subjects. Atherosclerosis, 2003; 166:303-309

7) Choi CU, Park EB, Suh SY, Kim JW, Kim EJ, Rha SW, Seo HS, Oh DJ, Park CG: Impact of aortic stiffness on cardiovascular disease in patients with chest pain: assessment with direct intra-arterial measurement. Am J Hypertens, 2007; 20:1163-1169

8) Yamashina A, Tomiyama H, Arai T, Hirose K, Koji Y, Hirayama Y, Yamamoto Y, Hori S: Brachial-ankle pulse wave velocity as a marker of atherosclerotic vascular damage and cardiovascular risk. Hypertens Res, 2003; 26:615-622

9) Tomiyama H, Hirayama Y, Hashimoto H, Yambe M, Yamada J, Koji Y, Motobe K, Shiina K, Yamamoto Y, Yamashinai A: The effects of changes in the metabolic syndrome detection status on arterial stiffening: a prospective study. Hypertens Res, 2006; 29:673-678

10) Miyaki K, Hara A, Naito M, Naito T, Nakayama T: Two new criteria of the metabolic syndrome: prevalence and the association with brachial-ankle pulse wave velocity in Japanese male workers. J Occup Health, 2006; 48:134140

11) Tsubakimoto A, Saito I, Mannami T, Naito Y, Nakamura S, Dohi Y, Yonemasu K: Impact of metabolic syndrome on brachial-ankle pulse wave velocity in Japanese. Hypertens Res, 2006; 29:29-37

12) Kim YK: Impact of the metabolic syndrome and its components on pulse wave velocity. Korean J Intern Med, 2006; 21:109-115

13) Lin CC, Liu CS, Li TC, Chen CC, Li CI, Lin WY: Microalbuminuria and the metabolic syndrome and its components in the Chinese population. Eur J Clin Invest, 2007; 37:783-790

14) Lin CC, Liu CS, Lai MM, Li CI, Chen CC, Chang PC, Lin WY, Lee YD, Lin T, Li TC: Metabolic syndrome in a Taiwanese metropolitan adult population. BMC Public Health, 2007; 7:239

15) Metz CE: Basic principles of ROC analysis. Semin Nucl Med, 1978; 8:283-298

16) Van der Schouw YT, Verbeek AL, Ruijs JH: ROC curves for the initial assessment of new diagnostic tests. Fam 
Pract, 1992; 9:506-511

17) Stehouwer CD, Henry RM, Ferreira I: Arterial stiffness in diabetes and the metabolic syndrome: a pathway to cardiovascular disease. Diabetologia, 2008; 51:527-539

18) Yokoyama H, Kuramitsu M, Kanno S, Tada J, Yokota $Y$, Kamikawa F: Relationship between metabolic syndrome components and vascular properties in Japanese type 2 diabetic patients without cardiovascular disease or nephropathy. Diabetes Res Clin Pract, 2007; 75:200-206

19) Sipilä K, Koivistoinen T, Moilanen L, Nieminen T, Reunanen A, Jula A, Salomaa V, Kaaja R, Kööbi T, Kukkonen-Harjula K, Majahalme S, Kähönen M: Metabolic syndrome and arterial stiffness: the Health 2000 Survey. Metabolism, 2007; 56:320-326

20) Achimastos AD, Efstathiou SP, Christoforatos T, Panagiotou TN, Stergiou GS, Mountokalakis TD: Arterial stiffness: determinants and relationship to the metabolic syndrome. Angiology, 2007; 58:11-20

21) Choi KM, Lee KW, Seo JA, Oh JH, Kim SG, Kim NH, Choi DS, Baik SH: Relationship between brachial-ankle pulse wave velocity and cardiovascular risk factors of the metabolic syndrome. Diabetes Res Clin Pract, 2004; 66:57-61
22) Ferreira I, Boreham CA, Twisk JW, Gallagher AM, Young IS, Murray LJ, Stehouwer CD: Clustering of metabolic syndrome risk factors and arterial stiffness in young adults: the Northern Ireland Young Hearts Project. J Hypertens, 2007; 25:1009-1020

23) Nakanishi N, Shiraishi T, Wada M: Brachial-ankle pulse wave velocity and metabolic syndrome in a Japanese population: the Minoh study. Hypertens Res, 2005; 28:125-131

24) Lin WY, Yao CA, Wang HC, Huang KC: Impaired lung function is associated with obesity and metabolic syndrome in adults. Obesity (Silver Spring), 2006; 14:16541661

25) Eckel RH, Grundy SM, Zimmet PZ: The metabolic syndrome. Lancet, 2005; 365:1415-1428

26) Nesto R: C-reactive protein, its role in inflammation, Type 2 diabetes and cardiovascular disease, and the effects of insulin-sensitizing treatment with thiazolidinediones. Diabet Med, 2004; 21:810-817

27) Rutter MK, Meigs JB, Sullivan LM, D'Agostino RB Sr, Wilson PW: C-reactive protein, the metabolic syndrome, and prediction of cardiovascular events in the Framingham Offspring Study. Circulation, 2004; 110:380-385 